Review

\title{
IFABP portal region insertion during membrane interaction depends on phospholipid composition
}

\author{
Eduardo de Gerónimo a,1, Luciana Rodriguez Sawicki a,1, Natalia Bottasso Arias ${ }^{\text {a }}$, Gisela Raquel Franchini ${ }^{\text {a }}$, \\ Fernando Zamarreño ${ }^{\mathrm{b}}$, Marcelo Daniel Costabel ${ }^{\mathrm{b}}$, Betina Córsico ${ }^{\mathrm{a}}$, Lisandro Jorge Falomir Lockhart ${ }^{\mathrm{a}, \mathrm{c}, *}$ \\ a Instituto de Investigaciones Bioquímicas de La Plata (INIBIOLP), CCT-La Plata (CONICET), Facultad de Cs. Médicas (UNLP), Calle 60 y 120,1900 La Plata, Argentina

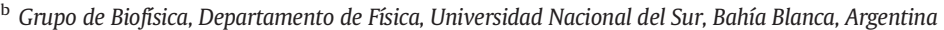 \\ c Laboratory of Cellular Dynamics, Max Planck Institute for Biophysical Chemistry, Am Fassberg 11, 37077 Göttingen, Germany
}

\section{A R T I C L E I N F O}

\section{Article history:}

Received 17 April 2013

Received in revised form 24 September 2013

Accepted 11 October 2013

Available online 19 October 2013

\section{Keywords:}

Fatty acid binding protein

Protein-lipid interaction

Intracellular lipid traffic

Intestinal lipid traffic

Brominated phospholipid

\begin{abstract}
A B S T R A C T
Intestinal fatty acid-binding protein (IFABP) is highly expressed in the intestinal epithelium and it belongs to the family of soluble lipid binding proteins. These proteins are thought to participate in most aspects of the biology of lipids, regulating its availability for specific metabolic pathways, targeting and vectorial trafficking of lipids to specific subcellular compartments. The present study is based on the ability of IFABP to interact with phospholipid membranes, and we characterized its immersion into the bilayer's hydrophobic central region occupied by the acyl-chains. We constructed a series of Trp-mutants of IFABP to selectively probe the interaction of different regions of the protein, particularly the elements forming the portal domain that is proposed to regulate the exit and entry of ligands to/from the binding cavity. We employed several fluorescent techniques based on selective quenching induced by soluble or membrane confined agents. The results indicate that the portal region of IFABP penetrates deeply into the phospholipid bilayer, especially when CL-containing vesicles are employed. The orientation of the protein and the degree of penetration were highly dependent on the lipid composition, the superficial net charge and the ionic strength of the medium. These results may be relevant to understand the mechanism of ligand transfer and the specificity responsible for the unique functions of each member of the FABP family.
\end{abstract}

(c) 2013 Elsevier B.V. All rights reserved.

\section{Introduction}

Intestinal fatty acid-binding protein (IFABP) belongs to a family of intracellular lipid binding proteins of low molecular mass (14-15 kDa) that bind long-chain fatty acids [1]. These proteins share an almost identical tertiary structure consisting of 10 antiparallel $\beta$-strands ( $\beta A$ to $\beta \mathrm{J}$ ) that form a $\beta$-barrel, which is capped by two short $\alpha$-helixes arranged as a helix-turn-helix segment located between $\beta A$ and $\beta B$ (Fig. 1A). It has been postulated that this helical domain is part of a "dynamic portal" that regulates fatty acid (FA) entry and exit from the internal binding cavity [2,3]. The precise physiological functions of these proteins in the cellular metabolism are as yet unclear, but it is hypothesized that they are important in intracellular transport

Abbreviations: 12AO, 12-(9-anthroyloxy)oleic acid; BrPC, brominated phosphatidylcholine; CL, cardiolipin; EPC, egg phosphatidylcholine; FA, fatty acid; FABP, fatty acid binding protein; LUV, large unilamellar vesicle; SUV, small unilamellar vesicle; Trp, tryptophan residue.

* Corresponding author at: Instituto de Investigaciones Bioquímicas de La Plata (INIBIOLP), Facultad de Cs. Médicas de La Plata, Calle 60 y 120 s/n, 1900 La Plata, Argentina.

E-mail addresses: Ifalomir@atlas.med.unlp.edu.ar, Ifalomi@gwdg.de

(L.J. Falomir Lockhart).

${ }^{1}$ These authors contributed equally to this work. and targeting of FA to specific membranous organelles and metabolic pathways.

IFABP is confined to enterocytes and, together with liver fatty acid binding protein (LFABP), represents a high proportion of the total cellular protein content [4]. Several differences between the two enterocyte FABPs, including ligand specificity, binding stoichiometry and ligand transport properties, suggest unique functional properties $[1,5,6]$. To deepen the transport function of the FABPs, we have used an in vitro fluorescence resonance energy transfer assay to examine the rate and mechanism of transfer of fluorescently tagged fatty acids from FABPs to phospholipid membranes. These studies have demonstrated that transfer of FAs from IFABP to membranes appears to occur during direct collisional interactions between the protein and the acceptor membrane [5]. In contrast, LFABP employs a different FA transfer mechanism, probably involving an initial release of the ligand to the aqueous milieu prior to its membrane association [7]. Employing mutant proteins, we have shown that the $\alpha$-helical domain of IFABP is responsible for the collisional mechanism of transfer $[2,8]$ and this involves charge-charge interactions with acidic domains on the membrane's surface, thereby facilitating the targeted transport of intracellular FA. Using a helixless variant of IFABP [9] and employing chimeric proteins generated by exchanging the helix-turn-helix domains between IFABP and LFABP, we have demonstrated that the $\alpha$-helical region of IFABP plays a primary role 
A

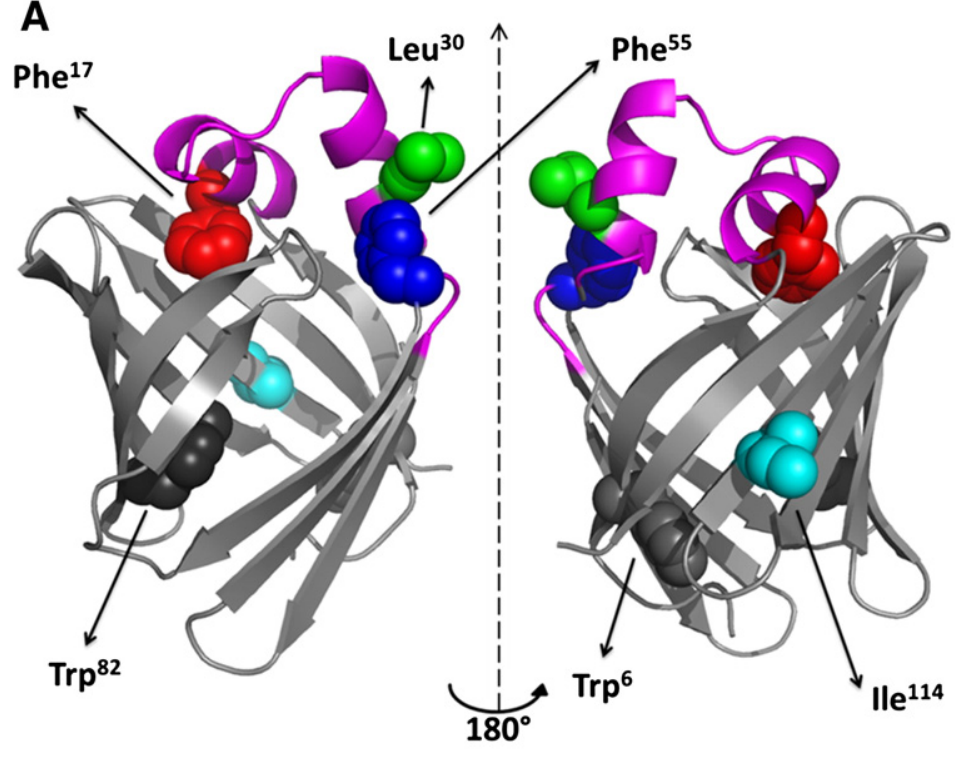

B
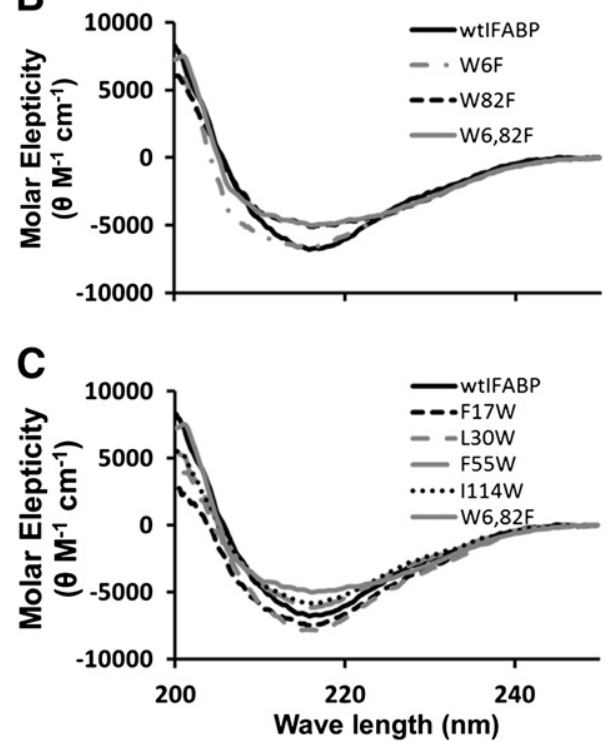

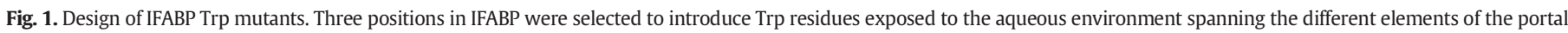

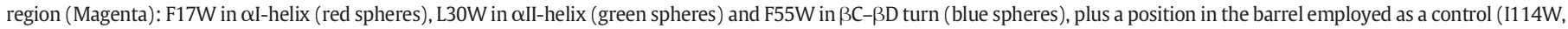

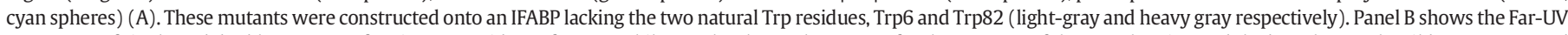

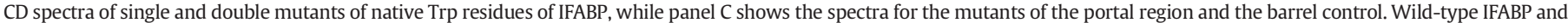
$\mathrm{W} 6,82 \mathrm{~F}$ template are shown in both cases as a reference, suggesting that all mutants fold properly.

in the collisional transfer of fatty acids from IFABP to membranes $[2,8]$ and that this domain determines the unique FA transfer mechanism from LFABP or IFABP to acceptor membranes.

With the aim of establishing FABPs' unique functions, we started a detailed analysis of the molecular determinants defining IFABP interaction with membranes. In our first work employing point mutants of IFABP, where positive charges of the lysine residues present in the $\alpha$-helical region were neutralized or reversed, we demonstrated that electrostatic interactions are a major determinant of the mechanism of FA transfer from IFABP to phospholipid membranes [10]. Molecular modeling studies of FABP-membrane interaction supported this idea and indicated that the $\alpha$-helical region of IFABP is energetically favored for its interaction with anionic membranes [11]. Moreover, the interaction of apo- and holo-IFABP with membranes was also analyzed employing a photoactivable reagent bound to membranes. These experiments showed that holo-IFABP interacts more strongly than the apo-form, probably indicating its capacity to selectively unload its ligand to the membranes, rather than uploading it from them [12].

As most of the enzymes involved in FA metabolism are membranebound, it is possible that IFABP could modulate lipid metabolic enzyme activity via direct interactions with membrane lipids and/or membrane proteins. For this reason, "collisional" FABPs are thought to be specific agents for targeting of FA selectively towards differential physiologically relevant targets (mitochondria, ER, lipid droplets, plasma membrane, etc.) [13]. The structural elements underlying the physical interaction of IFABP and membranes could have important physiological consequences, since they may dictate the FA trafficking pattern within a cell. The aim of the present work was set to characterize the interaction of IFABP with membranes employing IFABP Trp mutants as intrinsic probes. With this objective, a series of single Trp mutants spanning specific domains of the protein were engineered. We first characterized the degree of exposure of the different domains through the analysis of the fluorescence spectra of each Trp mutant, to determine the polar environment of each position; and the analysis of fluorescent quenching by acrylamide, which depends on solvent accessibility. We evaluated the Trp insertion into membranes by assessing its quenching by brominated phospholipids. We observed that the Trp residues from the portal region are immersed deep into the phospholipid bilayer. This interaction depended on the membrane composition and on the ionic strength of the media.

\section{Materials and methods}

\subsection{Materials}

12-(9-Anthroyloxy)-oleic acid (12AO) was purchased from Molecular Probes, Inc. (Eugene, OR). Egg phosphatidylcholine (EPC), N-(7-nitro-2,1,3-benzoxadiazol-4-yl) phosphatidylcholine (NBD-PC), bovine heart ardiolipin (CL), and brominated phosphatidylcholines (BrPCs) 1-palmitoyl-2-(6,7-dibromo)stearoyl-snglycero-3-phosphocholine (6,7-BrPC), 1-palmitoyl-2-(9,10-dibromo) stearoyl-sn-glycero-3-phosphocholine (9,10-BrPC) and 1-palmitoyl-2(11,12-dibromo)stearoyl-sn-glycero-3-phosphocholine (11,12BrPC) were obtained from Avanti Polar Lipids (Alabaster, AL). Acrylamide was purchased from Sigma-Aldrich (St. Louis, MI). Isopropyl- $\beta-D-$ thiogalactoside (IPTG) was obtained from Fisher (Fairlawn, NJ).

\subsection{Design of single tryptophan IFABP mutants}

The plasmid pET11d-rIFABP (carrying the cDNA of IFABP from Rattus norvegicus) was generously provided by Dr. Alan Kleinfeld and Dr. Ron Ogata (Medical Biology Institute, La Jolla CA). Since wild-type IFABP has two Trp residues buried inside of the binding site (W6 and W82), Phe was selected to replace them because it was assumed to be the less perturbing mutation from a structural point of view. We substituted alternatively one and the other Trp for Phe (W6F and W82F mutants), or both (W6,82F mutant). While the first ones were used as controls, the latter was used as the template for introducing single Trp residues at positions 17 (F17W), 30 (L30W), 55 (F55W) and 114 (I114W). The first three mutants correspond to the three segments involved in the portal region, namely the $\alpha$ I-helix, the $\alpha$ II-helix and the $\beta C-\beta D$ turn; and the mutant I114W in $\beta I$ was included as a control of the external face of the $\beta$-barrel. All single mutants were made by PCR megaprimer 
site-specific mutation [14], except for W6F that was generated as a single amplicon containing the full-length cDNA of the mutated protein. The double mutant W6,82F was prepared by digesting the single mutant W6F and W82F plasmids with Sall and Xbal (Promega) and then ligating the correct fragments. The same external primers were used for all PCR reactions: 5'-CGGATAACAATTCCCCTCTA-3' and 5'-TTCCTTTCGGGCTTT GTTAG-3'. All mutant cDNA sequences were subcloned into pET-11d using Xbal or NdeI (W6F) and BamHI restriction sites. All mutants were confirmed by sequence analysis. Table 1 shows the list of internal primers employed to generate each mutant.

\subsection{Protein expression and purification}

The recombinant proteins were overexpressed in Escherichia coli, harboring the pET11d-IFABP or the mutated versions of it, as detailed elsewhere $[2,5]$. The wild type and mutant proteins were purified from E. coli as described before [5]. Delipidation was carried out using a Lipidex-1000 column (Sigma). Purity was assessed in SDS-PAGE gels, and pure fractions were pooled and store at $-70^{\circ} \mathrm{C}$. Concentration was determined employing the individual extinction coefficients, determined according to Gill et al. [15].

\subsection{Vesicles preparation}

The desired proportions of different phospholipids were mixed in chloroform, dried under nitrogen and then lyophilized for at least $4 \mathrm{~h}$. Small unilamellar vesicles (SUVs) containing egg phosphatidylcholine (EPC) were prepared by sonication and ultracentrifugation as described previously [16,17]. For $12 \mathrm{AO}$ partitioning, $10 \mathrm{~mol} \%$ of NBD-PC was incorporated into the mixture of phospholipids, to serve as the fluorescent quencher of the anthroyloxy moiety. Large unilamellar vesicles (LUVs) were prepared at a concentration of $0.5 \mathrm{mM}$ total phospholipid by extrusion through polycarbonate membranes of $100 \mathrm{~nm}$ pore diameter (Avestin Inc., Ottawa, Canada) [12]. Vesicles composed of EPC, BrPC, EPC/BrPC (3:1 mol:mol), EPC/CL (3:1 mol: $\mathrm{mol})$ or $\mathrm{BrPC} / \mathrm{CL}(3: 1 \mathrm{~mol}: \mathrm{mol})$ were employed. Unless otherwise indicated, vesicles were prepared in $40 \mathrm{mM}$ Tris, $100 \mathrm{mM} \mathrm{NaCl}$ buffer (TBS), supplemented with $1 \mathrm{mM}$ EDTA in the case of vesicles containing $\mathrm{CL}$.

\subsection{Analysis of wild-type and mutant IFABP variants}

The conformation and integrity of the ligand binding site of the mutant proteins was examined by the following methods.

\subsubsection{Circular dichroism (CD)}

CD spectra were obtained at $25{ }^{\circ} \mathrm{C}$ on a J-810 model spectropolarimeter (Jasco) using a $0.1 \mathrm{~cm}$ path length quartz cuvette (Hellma). Spectra of wild-type and mutant proteins were obtained from five scans between 190 and $260 \mathrm{~nm}$ of an $18 \mu \mathrm{M}$ protein concentration solution in

Table 1

List of internal primers employed to generate Trp point mutants of IFABP.

\begin{tabular}{ll}
\hline Mutant name & ${\text { Internal } \text { primer }^{\text {a }}}^{\text {IFABP-W6F }}$ \\
\hline IFABP-W82F & 5'-CTACCATGGCATTTGATGGCACTTTCAAAGTAGACC-3' \\
IFABP-F17W & 5'-CCCATTTTCTCCATCCACTTTTCATAG-3' \\
IFABP-L30W & 5'-CATGAGCTCCCCACTTCCTCTTCAC-3' \\
IFABP-F55W & 5'-CATCAATGTTTCGCCAGTTGCTTGATTC-3' \\
IFABP-I114W & 5'-GGTAACGAACTATGGCAAACCTACAC-3' \\
\hline
\end{tabular}

a Underlined sequence indicates the bases mutated to eliminate or generate a Trp codon. a $25 \mathrm{mM}$ phosphate buffer (pH 7.4) [10]. Content of secondary structure was estimated as percentage of $\alpha$-helical, $\beta$-sheet and random coil with the online analysis software DichroWeb [18].

\subsubsection{Ligands relative affinity}

The partition coefficient $\left(K_{\mathrm{p}}\right)$ of $12 \mathrm{AO}$ between wild type or mutant IFABP and small unilamellar vesicles (SUVs) was determined by measuring $12 \mathrm{AO}$ fluorescence as a function of the molar ratio of protein/SUV. Briefly, a solution containing $5 \mu \mathrm{M}$ protein and $0.5 \mu \mathrm{M}$ $12 \mathrm{AO}$ in $40 \mathrm{mM}$ Tris, $100 \mathrm{mM} \mathrm{NaCl}, \mathrm{pH} 7.4$ (TBS) was titrated at $25^{\circ} \mathrm{C}$ with SUV containing 10\% NBD-PC instead an equivalent amount of EPC $[19,20] . K_{\mathrm{p}}$ is defined as:

$K_{\mathrm{p}}=\frac{\frac{[12 \mathrm{AO}: \mathrm{FABP}]}{[\mathrm{FABP}]}}{\frac{[12 \mathrm{AO}: \mathrm{SUV}]}{[\mathrm{SUV}]}}$

and was estimated by fitting the equation:

$F_{\text {rel }}=A \times \frac{K_{\mathrm{P}}}{K_{\mathrm{P}}+\frac{[\mathrm{SUV}]}{[\mathrm{FABP}]}}+B \times \frac{[\mathrm{SUV}]}{[\mathrm{FABP}]}$

to the data, where $F_{\text {rel, }}$ [SUV], [FABP], $A$ and $B$ are the relative fluorescence change, the molar concentration of SUVs, the protein concentration, and the fitting parameters, respectively. The fitting parameter $A$ represents the specific signal of $12 \mathrm{AO}$ bound to IFABP in the absence of vesicles; and $B$ the correction factor by the optical disturbance due to the presence of the vesicles.

\subsection{Fluorescence spectroscopy}

Fluorescence spectra were recorded on a Fluorolog 3 fluorimeter (Horiba/Jobin Yvon, UK). Trp fluorescence emission spectra were recorded from 300 to $400 \mathrm{~nm}$, with excitation wavelength set at $295 \mathrm{~nm}$ to prevent excitation of the tyrosine residues. The slit width was set to $5 \mathrm{~nm}$ for both the excitation and the emission. The protein concentration employed was $5.0 \mu \mathrm{M}$. For the quenching experiments, acrylamide was added from a $5.0 \mathrm{M}$ stock solution to the protein solution from 0 to $400 \mathrm{mM}$, and LUVs were added from a $0.5 \mathrm{mM}$ stock. After each addition, the samples were equilibrated for $3 \mathrm{~min}$ at $25{ }^{\circ} \mathrm{C}$ before the spectrum was recorded. Fluorescence quenching experiments were analyzed by the Stern-Volmer plots, and the curves were used to fit the modified form of the Stern-Volmer equation,

$\frac{F_{0}}{F}=1+K_{\mathrm{SV}} \times[\mathrm{Q}]$

where $F_{0}$ is the fluorescence intensity in the absence of quencher, $K_{\mathrm{SV}}$ is the Stern-Volmer constant, $[Q]$ is the molar concentration of quencher that gives a fluorescence intensity $F$.

\subsection{Estimation of membrane insertion depth of tryptophan residues}

Brominated phospholipids, in which bromine atoms are located at different positions in the acyl chains, have been used as effective Trp fluorescence quenching agents [21] without a significant perturbation of the membrane bilayer's properties [22]. The quenching is sensitive enough to calculate the distance of Trp residues to the center of the phospholipid bilayer by the distribution analysis method [23] when they are located within the region of the acyl chains. This method of analysis has been shown to be more appropriate than the Parallax calculation [21] for systems where multiple configurations are possible or complete knowledge of the behavior of the system studied is not possible. Therefore, to estimate the average Trp insertion depth, Br-depth depending quenching of Trp fluorescence results were 
employed to fit a Gaussian distribution of Trp distance to the center of the bilayer (Eq. (4)):

$\operatorname{Ln}\left(\frac{F_{0}}{F_{h}}\right)=\frac{S / c}{\sigma \sqrt{2 \pi}} e^{-\frac{\left(h-h_{m}\right)^{2}}{2 \sigma^{2}}}$

where $F_{0}$ and $F_{\mathrm{h}}$ are the fluorescence intensities in the absence and presence of the quencher at a distance $h$ from the center of the bilayer after equilibration of the protein-LUV mixtures. The fitting parameters $h_{\mathrm{m}}, \sigma$ and $S$ correspond to the mean depth of the Trp residue, the dispersion and the area of the Gaussian function, respectively. The parameter $c$ corrects the quenching efficiency by the concentration of the quencher in the phospholipid bilayer.

LUVs of BrPCs were prepared with either 100\% 6,7-BrPC, 9,10-BrPC or $11,12-\mathrm{BrPC}$, and a $5.0 \mu \mathrm{M}$ dilution of each IFABP's Trp mutants in TBS were titrated with increasing concentration of them, from 0 to $42 \mu \mathrm{M}$ BrPCs. After each addition, the sample was incubated at $25^{\circ} \mathrm{C}$ for $3 \mathrm{~min}$ to equilibrate before recording the emission spectrum of Trp. The depth of immersion was calculated using the 6,7- and 9,10-BrPC and 11,12-BrPC pairs to fit Eq. (4). To analyze the dependence of the depth penetration on the composition of the vesicles, we repeated the analysis employing vesicles with 25 mol\% of CL (25\% CL-75\% BrPC $\mathrm{mol} / \mathrm{mol}$ ). In this case, LUVs composed of $25 \mathrm{~mol} \%$ of EPC (25\% EPC$75 \% \mathrm{BrPC}$ ) were used as controls. The effect of the ionic strength on the protein-membrane interaction was assessed by varying the $\mathrm{NaCl}$ concentration from 100 to $1000 \mathrm{mM}$ in the TBS during the preparation of the LUVs.

\subsection{Molecular modeling}

Atomic coordinates for the membrane were snapshots generated computationally and equilibrated by molecular dynamics procedures [24]. As a single component of the neutral membrane, we used POPC and anionic forms of membranes were developed with a mix of $75 \%$ POPC and 25\% CL. Structure of IFABP is based on that previously reported (PDB ID: 1IFB), and its relative positions were obtained taking into account experimental data, electrostatic free energy between the protein and the membrane, and protein structure conservation so that no deviations from currently available experimental geometries were induced. Figures were developed with PyMOL Molecular Graphics System (Schrödinger, LLC) [25].

\section{Results}

\subsection{Structural and ligand properties of IFABP's tryptophan mutants}

To examine whether the portal region of IFABP is the membrane binding domain, we undertook the construction of point mutants inserting Trp residues in the three different structural elements of the portal region: $\alpha$ I-helix, $\alpha$ II-helix and the $\beta C-\beta D$ turn [3]. After eliminating the naturally occurring Trp residues at positions 6 and 82, Trp residues were introduced separately in the $\alpha$ I-helix (F17W), the $\alpha$ II-helix (L30W) and the $\beta$ C- $\beta$ D turn (F55W) (Fig. 1A). An extra Trp residue was incorporated at the $\beta$ I strand (I114W) to serve as a control of the $\beta$-barrel. To control for potential alterations in the overall folding of the point mutants, $\mathrm{CD}$ spectroscopy was used to evaluate their secondary structure. The far-UV CD spectra of IFABP showed a maximum at 198-200 nm and a minimum near $215 \mathrm{~nm}$, typical of proteins with predominant $\beta$-structures, and its shape and intensity agreed with previously published data $[6,10,26]$. Fig. 1B and $C$ shows the far-UV CD spectra for all mutants employed in this study plus the one of IFABP-W6,82F employed as a template for constructing the single-Trp mutants. Minor changes in the secondary structure of mutants W6F, W82F and W6,82F can be observed (Fig. 1B). Also, no significant differences are detectable in the Trp mutants compared to the wild type IFABP or the W6,82F mutant (Fig. 1C). The $\alpha, \beta$ and random-coil content estimations for each variant are presented in Table 2.

The relative partition coefficient $\left(K_{\mathrm{P}}\right)$ of $12 \mathrm{AO}$ between the IFABP variants and zwitterionic SUVs (EPC 90\%, NBD-PC 10\%) was determined in order to evaluate the integrity and functionality of the ligand binding site of the Trp mutants. All mutants showed a similar behavior, a progressive decrease of $12 \mathrm{AO}$ fluorescence as a function of increasing NBD-PC-containing vesicles' concentration, indicating the ability of each mutant to transfer $12 \mathrm{AO}$ to acceptor SUVs vesicles. Furthermore, the results of $K_{\mathrm{P}}$ presented in Table 2 fall within the 95\% confidence that includes the value of the native IFABP and indicate a preferential partitioning of $12 \mathrm{AO}$ to SUVs, also in agreement with previous results $[2,6,10]$. These results indicate that the overall folding of the protein remains intact and that, even though two residues of the hydrophobic core were mutated (W6F and W82F), the relative affinity of the mutant proteins for fluorescent fatty acid analogs is essentially the same as in wild-type IFABP.

Altogether, these controls suggest that no major alterations were induced in the overall conformation or the properties of the binding site in the Trp-mutants of IFABP compared to the wild-type protein. Our results agree with previous reports of Trp to Phe mutations that did not alter the native folding or ANS binding of IFABP [26].

\subsection{Analysis of fluorescence spectra of tryptophan residues}

The fluorescence emission spectrum of tryptophan reports on its immediate environment around it. Trp residues in a polar aqueous environment show an emission maximum near 340-350 nm, whereas Trp residues in an apolar environment present a blue-shifted emission. IFABP has two Trp residues located at positions 6 and 82. $\operatorname{Trp}^{6}$ constitutes part of the hydrophobic core that drives the folding of the protein and is completely hidden from the aqueous environment

Table 2

Structural controls of Trp mutants of IFABP.

\begin{tabular}{|c|c|c|c|c|c|c|c|}
\hline \multirow[t]{2}{*}{ Protein } & \multirow{2}{*}{$\begin{array}{l}\varepsilon^{280 \mathrm{~nm}} \\
\left(\mathrm{M}^{-1} \mathrm{~cm}^{-1}\right)\end{array}$} & \multicolumn{4}{|l|}{$\mathrm{CD}$ spectroscopy ${ }^{\mathrm{a}}$} & \multirow[t]{2}{*}{ Fluorescence maximum (nm) } & \multirow[t]{2}{*}{$K_{\mathrm{P}}\left(\left[\right.\right.$ SUV] $/[\text { Protein] })^{\mathrm{b}}$} \\
\hline & & Minimum (nm) & $\% \alpha$ & $\% \beta$ & $\% \mathrm{rc}$ & & \\
\hline wtIFABP & 16,000 & 216 & 10 & 40 & 28 & 329.5 & $0.13 \pm 0.04$ \\
\hline W6F $\left(\operatorname{Trp}^{82}\right)$ & 10,816 & 215 & 10 & 35 & 32 & 332 & $0.06 \pm 0.03$ \\
\hline W82F $\left(\operatorname{Trp}^{6}\right)$ & 10,816 & 217 & 6 & 42 & 27 & 324.5 & $0.08 \pm 0.03$ \\
\hline W6,82F & 5120 & 216 & 3.5 & 41 & 32 & $N A^{c}$ & $N^{d}$ \\
\hline F17W & 10,816 & 216 & 8 & 33 & 35 & 338 & $0.17 \pm 0.09$ \\
\hline L30W & 10,817 & 216 & 13 & 38 & 31 & 340 & $0.16 \pm 0.04$ \\
\hline F55W & 10,816 & 216 & 6 & 42 & 30 & 341 & $0.21 \pm 0.05$ \\
\hline I114W & 10,817 & 216 & 6 & 40 & 32 & 340 & $0.16 \pm 0.07$ \\
\hline
\end{tabular}

a CD calculations of secondary structure were performed employing DichroWeb online software. rc: Random coil. The unaccounted content of secondary structure to reach $100 \%$ should be considered as turns.

b $K_{\mathrm{sv}}$ results are average of at least 3 independent measurements \pm SEM.

c NA: not applicable.

d ND: not determined. 

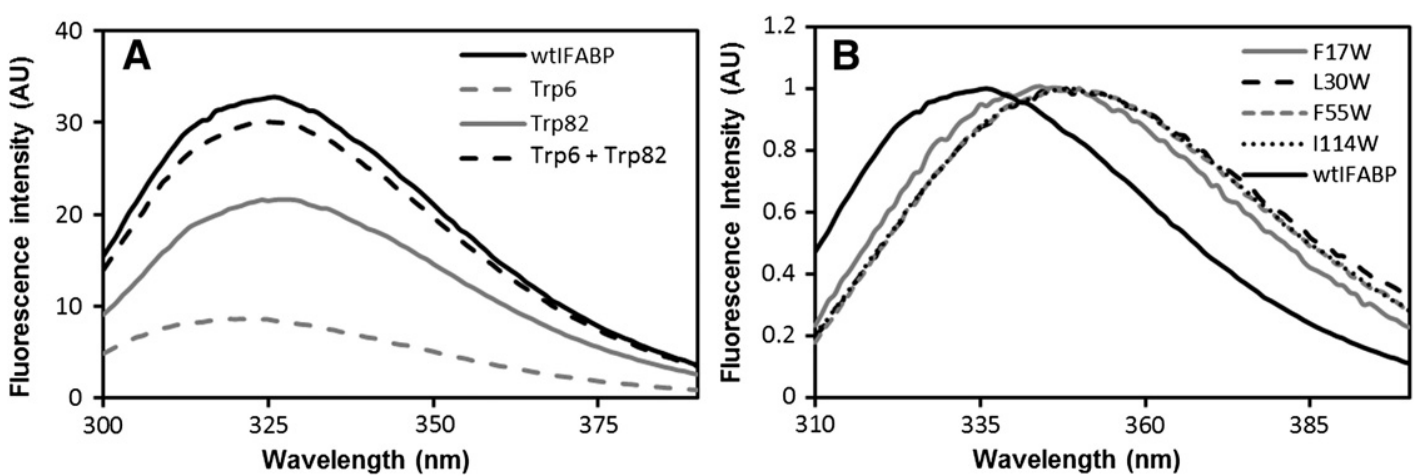

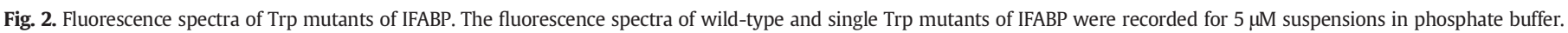

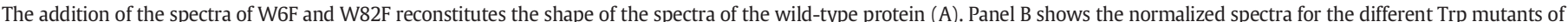
IFABP employed in the following assays.

while $\operatorname{Trp}^{82}$ is immersed in a distinct environment forming part of the bottom of the ligand binding site, according to the crystal structure PDB-ID: 1IFB [27]. The fluorescence spectra of mutants W6F $\left(\operatorname{Trp}^{82}\right)$ and W82F $\left(\operatorname{Trp}^{6}\right)$ indicate that both Trp residues show a maximum fluorescence emission at wavelengths expected for a Trp located in apolar environments, but their environments are not equivalent. $\operatorname{Trp}^{6}$ showed a slightly more shifted spectrum to shorter wavelengths $\left(\lambda_{\max }=324 \mathrm{~nm}\right)$ compared to that of $\operatorname{Trp}^{82}\left(\lambda_{\max }=332 \mathrm{~nm}\right)$ as shown in Fig. 2A and Table 2. This could be explained by the fact that $\operatorname{Trp}^{6}$ is surrounded by a more hydrophobic environment; while $\operatorname{Trp}^{82}$ is accessible from the binding cavity and next to $\operatorname{Arg}^{106}$, the anchor residue that forms a salt bridge with the carboxylic head of the fatty acids when the ligand is bound to the protein. Furthermore, the binding cavity may be filled with water located close to $\operatorname{Trp}^{82}$ in the apo-form, and partially responsible for a more red-shifted spectrum than W82F.
Noteworthy, the addition of the fluorescence spectra of mutants W6F and W82F reconstitutes the spectra of the native IFABP, further proving that the mutations introduced to neutralize the original Trp residues do not affect the average folding of the protein in solution, or, particularly in this case, the immediate environment of the other natural Trp residue.

The steady-state fluorescence spectra of the portal mutants showed a red-shifted emission spectrum compared to the wild-type protein, but with significantly lower quantum yield (not shown), as expected for water exposed fluorescent probes. The barrel control I114W also showed a red shifted emission, but the signal was approximately double than for the portal region mutants. Mutants L30W, F55W and I114W showed a maximum emission around $340 \mathrm{~nm}$, while for $\mathrm{F} 17 \mathrm{~W}$ the maximum was $\lambda_{\max }=338 \mathrm{~nm}$ (Fig. $2 \mathrm{~B}$ and Table 2). This could be explained by the orientation of the original $\mathrm{Phe}^{17}$ in the crystal

\section{Free in buffer}
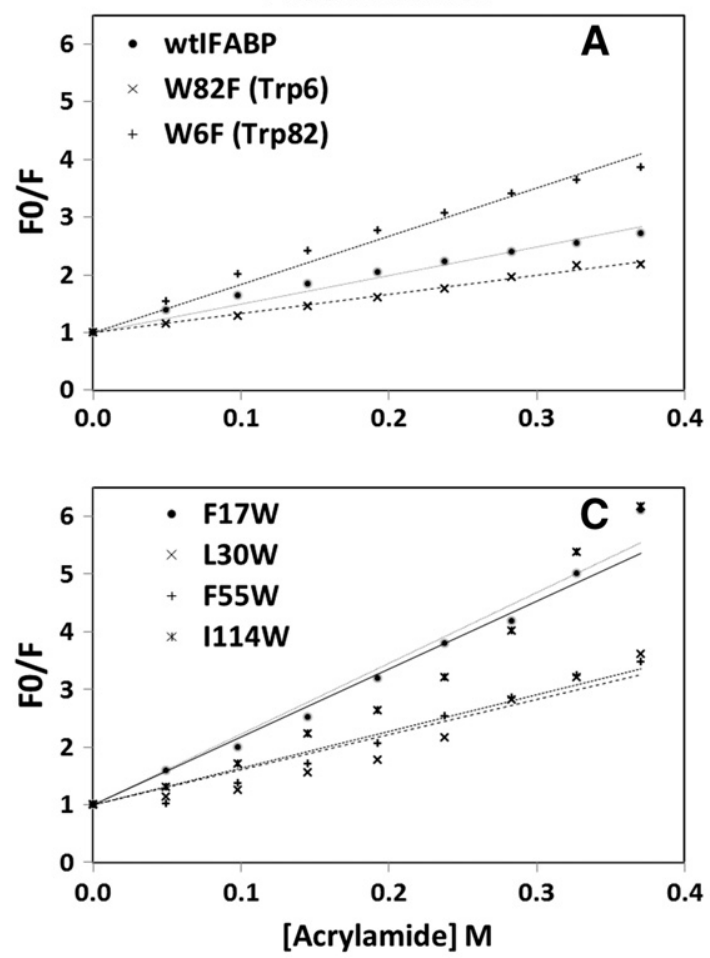

\section{$+40 \mu \mathrm{M}$ LUVs}
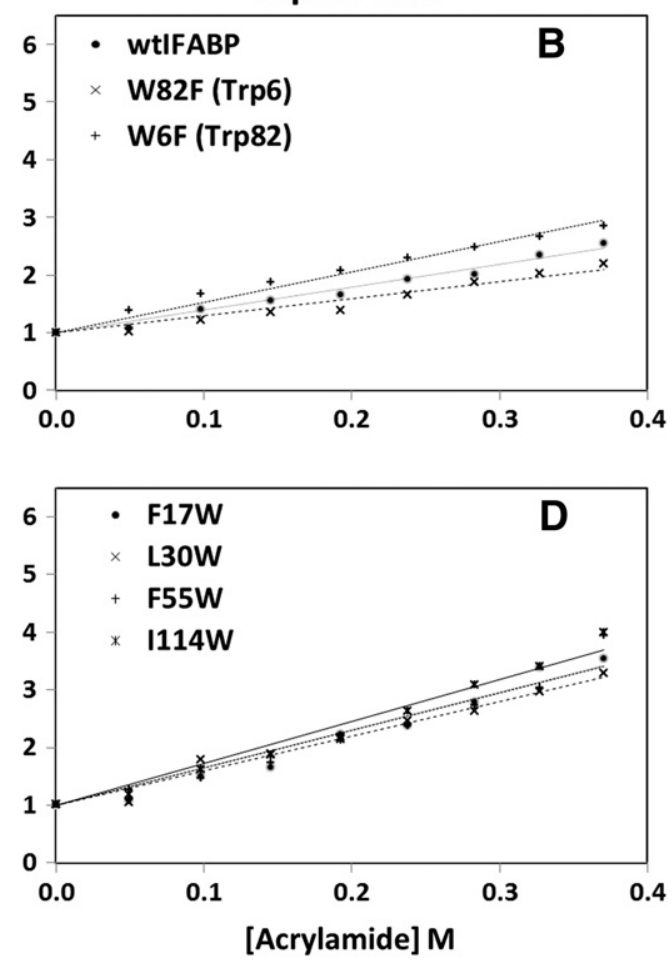

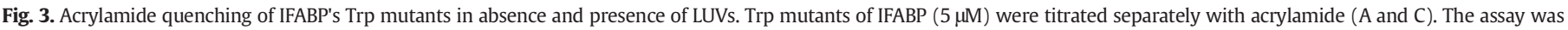

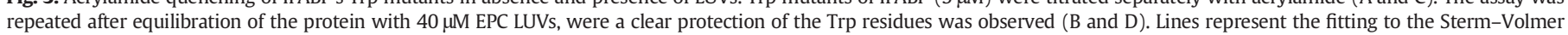
equation (Eq. (3)). Plots represent a representative titration of a total of at least three replicates. Final results of $K_{\mathrm{SV}}$ constants are presented in Table 3. 
structure, which is not completely exposed to the solvent (unlike $\mathrm{Leu}^{30}$, $\mathrm{Phe}^{55}$ or $\mathrm{Ile}^{114}$ ) and also is rather close to two other hydrophobic residues, Phe $^{117}$ and Phe $^{119}$.

\subsection{Acrylamide quenching}

We analyzed the ability of acrylamide to quench the Trp fluorescence of the IFABP mutants by titrating them in the presence and absence of EPC-LUVs. Quenching by acrylamide is mainly a collisional effect and is thus dependent on the concentration of quencher and the solvent accessibility of the Trp residue. Fig. 3 shows the Stern-Volmer plots obtained for the wild-type protein and its mutants. The results of SternVolmer constants derived from the fitting of Eq. (3) to these results are summarized in Table 3.

The $K_{\mathrm{SV}}$ obtained by quenching in aqueous solution show that the native $\operatorname{Trp}^{6}$ residue of IFABP is considerably more protected from the environment compared with the $\operatorname{Trp}^{82}$ or those inserted in the portal region, in agreement with the observed blue-shifted emission. The larger exposure of the $\operatorname{Trp}^{82}$ compared with the $\operatorname{Trp}^{6}$ is probably due to access of the soluble quencher to the empty binding cavity. I114W and F17W Trp mutant were more easily quenched by Acrylamide $\left(K_{\mathrm{SV}}=12.3 \pm\right.$ 1.5 and $12.5 \pm 1.3$, respectively) than $\mathrm{F} 55 \mathrm{~W}(7.1 \pm 0.6)$ and $\mathrm{L} 30 \mathrm{~W}$ $(6.9 \pm 0.3)$, correlating largely with the emission maximum (Table 2). The unexpectedly high exposure of the $\operatorname{Trp}^{17}$ could be explained by the acrylamide access from the empty binding site. The effect of the addition of a 1:1 proportion of oleic acid to protein before the titration with acrylamide was different on F17W and I114W (that were partially protected) than for L30W and F55W (that were more exposed by the presence of the ligand). This could be explained by the entry of the ligand into the binding pocket that also slightly expels residues 30 and 55 towards the aqueous medium. These changes correlate with the subtle conformational changes between apo- and holo-forms of IFABP $[27,28]$.

We also evaluated the capacity of LUVs in the sample to protect the Trp residues from the acrylamide quenching. A protection factor was calculated as the ratio between the $K_{\mathrm{SV}}$ obtained for the free in solution and in the presence of membrane or the oleic acid bound proteins forms (Table 3). Binding to $100 \%$ EPC vesicles induces the protection of Trp residues at positions 82,17 and 114 , a less evident protection was sensed by the Trp in position 55, whereas Trp at positions 6 and 30 do not seem to be affected. The protection of Trp at position 114 after incubation with vesicles could be explained by a conformational change of the $\beta$-barrel, or by a side interaction of the protein.

\subsection{Membrane penetration depth of IFABP portal domain}

Depth-dependent fluorescence quenching of IFABP Trp mutants was studied using LUV prepared with phospholipids dibrominated at different depths. The degree of membrane penetration of Trp residues located in different regions of IFABP was estimated by analyzing the relative quenching effect of the different brominated lipids on Trp fluorescence. Noteworthy, the synthetic brominated PC derivatives employed for this study, which do not contain double bonds, form bilayers of equal thickness ( $49 \AA$ ) and similar properties, comparable to lipids with a single unsaturated bond $[29,30]$.

The results obtained for the different Trp mutants are presented in Fig. 4. The titration curves clearly deviate from a straight line. Unlike acrylamide quenching, which is a diffusion-mediated process, in the case of quenching by brominated phospholipids the plots report the thermodynamic equilibrium of the protein distributed between the free and LUV-bound states, instead of the accessibility of the quencher to the fluorophore.

The incubation of the single mutants with the native Trp residues with BrPC LUVs showed small changes on Trp fluorescence. $\operatorname{Trp}^{82}$ (W6F), in particular, showed no changes in Trp fluorescence when $\mathrm{Br}$ atoms were at positions 9 or deeper, but for vesicles with $\mathrm{Br}$ atoms at positions 6 and 7 a small but consistent increase in its fluorescence was actually recorded compared with the fluorescent signal of the protein free in solution. This unexpected effect could be suggesting that the protein-complex formation is accompanied by a conformational change of the ligand binding site and or the hydrophobic core, increasing the quantum yield of $\operatorname{Trp}^{82}$. On the other hand, $\operatorname{Trp}^{6}$ (W82F) showed a small, but non-significant, decrease in Trp fluorescence when titrated with brominated LUVs. Furthermore, no difference was observed between vesicles with $\mathrm{Br}$ atoms located at different heights. In all these cases, changes were lower than $8 \%$ of the initial fluorescence, compatible with the results expected for residues that are not close to the membrane's hydrophobic core.

On the other hand, Trp mutants of the portal region of IFABP showed a distinct behavior depending on the residue and the quencher position. All Trp residues exposed to the solvent were quenched on a concentration dependent manner by brominated LUVs, but $\operatorname{Trp}^{17}$ (F17W) and Trp5 (F55W) showed a larger effect when incubated with 6,7-BrPC containing LUVs than with those with $9,10-\mathrm{BrPC}$ or 11,12 -BrPC that were almost undistinguishable (around 18\% at the largest concentration tested, $40 \mu \mathrm{M})$. Furthermore, $\operatorname{Trp}^{17}$ suffered a larger quenching effect $(-35 \%)$ with 6,7BrPC LUVs than $\operatorname{Trp}^{55}$ (-29\%). In the case of $\operatorname{Trp}^{30}$ (L30W), 6,7BrPC and 9,10-BrPC LUVs had near equivalent effects $(-22 \%)$, while $11,12-B r P C$ LUVs showed a slightly smaller effect $(-15 \%)$. These results suggest that the three Trp inserted in the portal region seem to be, at least transiently, located into the phospholipid membrane's hydrophobic core, and that $\operatorname{Trp}^{30}$ could be closer to the medium plane of the bilayer. Finally, the incubation of the control mutant with the Trp residue incorporated in the outer side of the $\beta$-barrel (I114W) showed equivalent effects with all brominated LUVs and a quenching effect intermediate between that observed for the portal region mutants and the native Trp residues (around $-15 \%$ ), suggesting that it may be closer to the phospholipid interface than the natural Trp residues, but it did not get close enough to the hydrophobic core to detect any specific quenching by the Br atoms at different positions for this type of vesicles.

Table 3

Acrylamide quenching of native IFABP and single Trp mutants in the presence and absence of oleic acid and EPC LUVs.

\begin{tabular}{|c|c|c|c|c|c|}
\hline \multirow[t]{3}{*}{ Protein } & \multirow{3}{*}{$\frac{\text { Buffer }}{\mathrm{K}_{\mathrm{SV}}{\text { (acrylamide })^{\mathrm{a}}}^{\mathrm{a}}}$} & \multicolumn{2}{|l|}{ EPC LUVs } & \multicolumn{2}{|l|}{ Oleic Acid ${ }^{\mathrm{b}}$} \\
\hline & & \multirow{2}{*}{$\begin{array}{l}K_{\text {SV }} \\
\text { (acrylamide) }^{\mathrm{a}}\end{array}$} & Protection factor & \multirow{2}{*}{$\begin{array}{l}K_{\mathrm{SV}} \\
\text { (acrylamide) }^{\mathrm{a}}\end{array}$} & \multirow{2}{*}{$\frac{\text { Protection factor }}{\left(K_{\mathrm{SV}} \text { ratio, buffer } / \mathrm{OA}\right)}$} \\
\hline & & & ( $K_{\mathrm{SV}}$ ratio, buffer/LUVs) & & \\
\hline wtIFABP & $4.4 \pm 0.4$ & $4.7 \pm 0.3$ & 0.94 & $2.5 \pm 0.3$ & 1.72 \\
\hline W6F $\left(\operatorname{Trp}^{82}\right)$ & $7.7 \pm 1.1$ & $5.1 \pm 1.3$ & 1.51 & $5.5 \pm 0.5$ & 1.40 \\
\hline W82F $\left(\operatorname{Trp}^{6}\right)$ & $3.5 \pm 0.3$ & $3.3 \pm 0.5$ & 1.03 & $4.9 \pm 0.02^{c}$ & 0.71 \\
\hline F17W & $12.5 \pm 1.3$ & $7.7 \pm 0.4$ & 1.62 & $7.8 \pm 0.8$ & 1.60 \\
\hline L30W & $6.9 \pm 0.3$ & $6.7 \pm 0.7$ & 1.03 & $12.9 \pm 1.9$ & 0.53 \\
\hline F55W & $7.1 \pm 0.6$ & $6.0 \pm 0.7$ & 1.19 & $10.7 \pm 0.3^{\mathrm{c}}$ & 0.66 \\
\hline I114W & $12.3 \pm 1.5$ & $8.1 \pm 0.1$ & 1.52 & $9.8 \pm 1.0$ & 1.25 \\
\hline
\end{tabular}

${ }^{\mathrm{a}} K_{\mathrm{SV}}$ results are average of at least 3 independent measurements \pm SEM.

b A 1:1 ratio of FABP and fatty acid was employed.

c Only 2 determinations were made in these cases. 

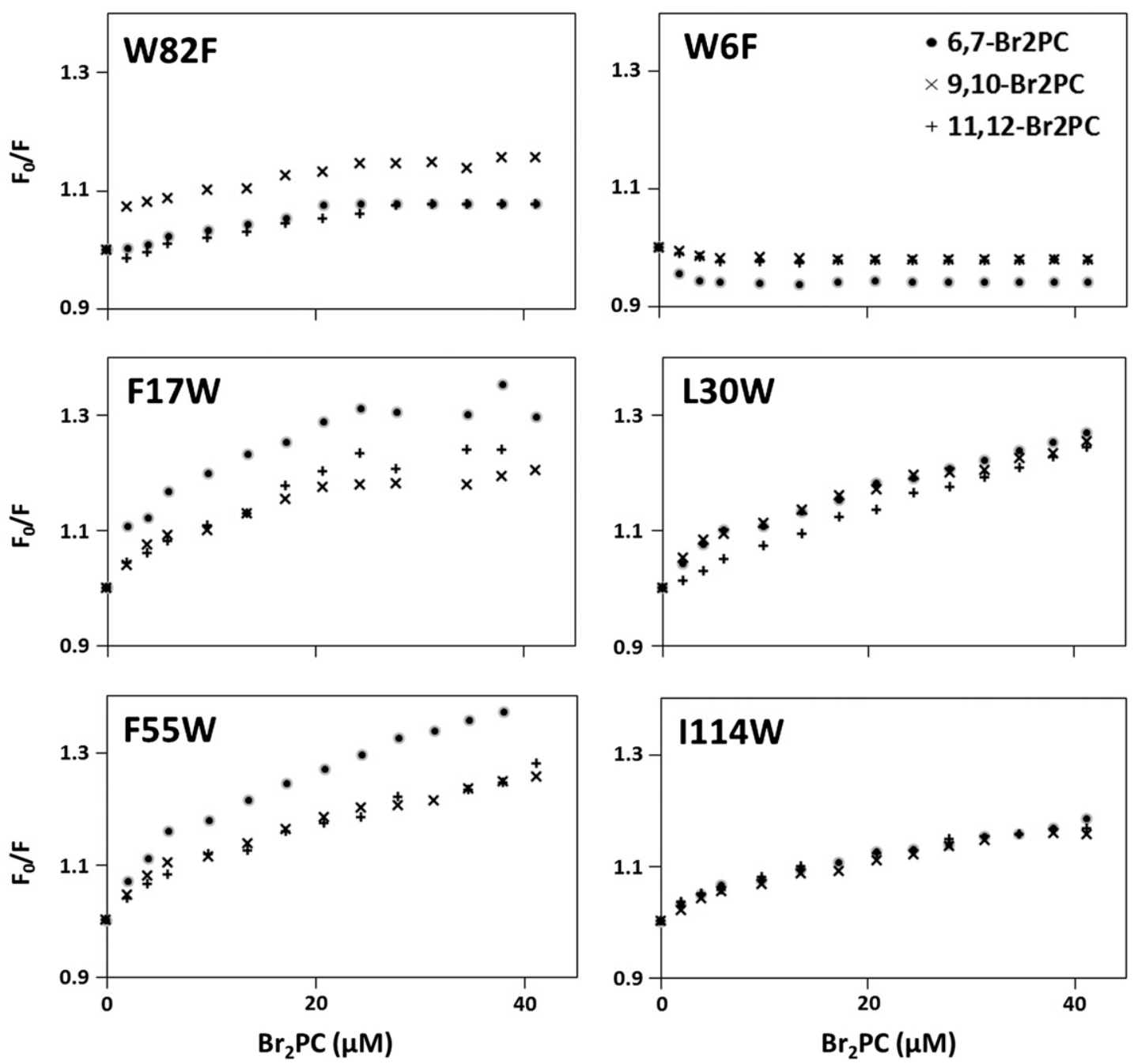

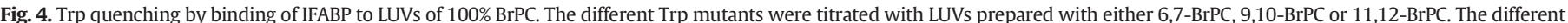

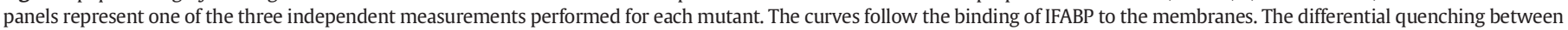
the different types of vesicles were employed to fit the parameters of Eq. (4) presented in Table 4.

All together these results suggest that the different components of the portal region are participating with different degrees of immersion in the bilayer, while the natural Trp residues of the hydrophobic core and the control mutant are not in close contact with the membrane.

The distances of the Trp residues to the center of the bilayer were estimated based on Eq. (4) comparing the differential quenching effect of the $\mathrm{Br}$ atoms located at different positions in the acyl chains of PC. This method detects lower quenching effects for IFABP, compared to transmembrane peptides, probably due to the fact that IFABP is a soluble protein and only is inserted partially and transiently into the membrane. Therefore, calculations were performed only for the mutants that showed more than $20 \%$ quenching for at least one type of vesicles at $40 \mu \mathrm{M}$ LUVs. For the other cases, calculated distances were larger than $20 \AA$, indicating that the Trp residues were distributed mainly outside the membrane, where the method shows no sensitivity. Thus, it was not possible for native Trp residues to estimate a distance from the central plane of the membrane. The same was true for the Trp at position 114 in the case of $100 \% \mathrm{Br}_{2}$-PC LUVs. We also studied the dependency of Trp insertion on the lipid composition of the vesicles. The results for Trp of the portal region for all types of vesicles tested are shown in Fig. 5 and in Table 4. These results show that Trp residues located in the portal region get in contact with the membrane's hydrophobic core when incubated with brominated phospholipids. Nevertheless, only L30W is deeply immersed between the acyl chains, as judged by the narrow distribution with a maximum at a shorter distance from the center of the bilayer. Concurrently, F17W and F55W stay between the polar head-groups and the hydrophobic central portion of the membrane. This is also confirmed by the wider distributions, observed for residues 17 and 55 compared to L30W, indicating the lower precision in the estimations of their positions. Trp residues in other positions seem to be located outside the membrane when incubated with $100 \% \mathrm{BrPC}$.

\subsection{Modulation of membrane interaction and Trp residues insertion}

Previous reports indicated that electrostatic forces between IFABP and model lipid membranes drive their interaction, and that this is strongly affected by the surface charge of the vesicles (phospholipid composition) and the ionic strength of the environment [10-12,31]. Cardiolipin, in particular, seems to be a significant and unique interacting partner for IFABP, potentially involved in vectorial transfer of its cargo towards the mitochondria $[2,10,12]$. Therefore, we analyzed how these factors modulate Trp residues insertion into the membranes for IFABP's Trp mutants by BrPC dependent quenching.

BrPC LUVs containing either 25\% cardiolipin (CL) (25\% CL, 75\% $\mathrm{BrPC}$ ) or $25 \%$ egg phosphatidylcholine (EPC) (25\% EPC, 75\% BrPC) were employed to analyze the dependence of Trp insertion on lipid composition [32]. Results presented in Fig. 5 suggest that the change in lipid composition for CL allows a deeper insertion of the protein as 
A

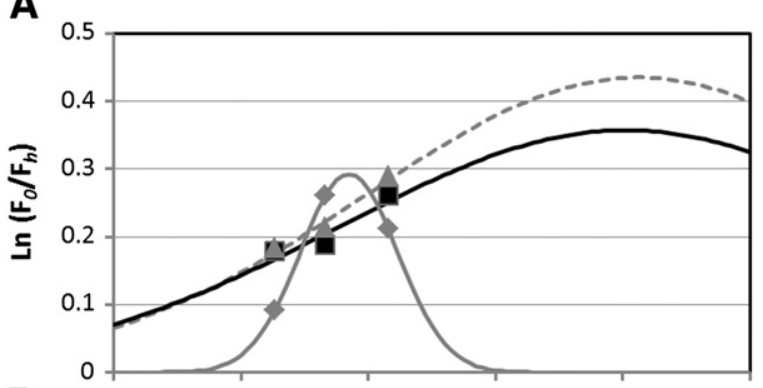

B

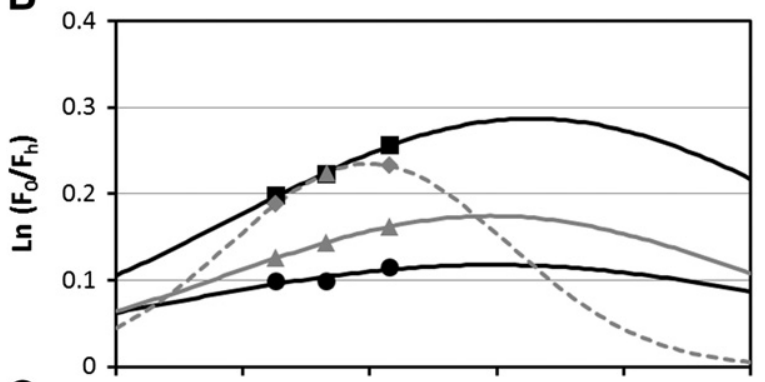

$c$

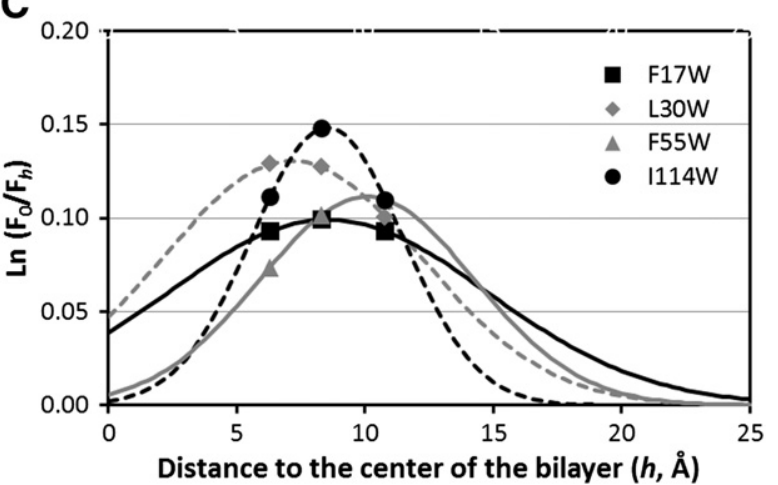

Fig. 5. Distribution of Trp location in phospholipid bilayers. Trp residues of mutant IFABP were titrated with LUVs containing brominated phospholipids at different positions of their acyl chains. The differential quenching effect of each type of lipid was employed to calculate the depth of the Trp immersion into the membrane by fitting Eq. (4) to the data. The different panel show representative results obtained for $100 \%$ brominated LUVs (A), 75\% BrPC-25\%EPC (B) and 75\% BrPC-25\%CL (C). Fitted parameters are summarized in Table 4.

all Trp are largely quenched already in the presence of $25 \%$ natural zwitterionic lipids (EPC). This is probably due to the presence of multiple polyunsaturated acyl chains that define a more flexible bilayer. When $25 \%$ CL was employed instead of the EPC, the distances calculated indicate an even deeper immersion of all Trp residues of the portal region. This is consistent with the idea that the electrostatic interactions are important for IFABP-membrane approach and interaction, but also suggests that the protein orientation on the membrane interphase depends on the charged head-groups and, hence, on the phospholipid composition. A significant change in orientation of IFABP in the membrane complex is required for the $\beta$-barrel control (I114W) to be accommodated inside the bilayer along with the other Trp mutants when incubated with CL-containing vesicles. Another possibility, not necessary mutually exclusive, would be that IFABP may have multiple interaction modes when interacting with these anionic vesicles.

Finally, we analyzed the effect of increasing ionic strength on IFABP membrane interaction. IFABP Trp mutants were titrated with $100 \% \mathrm{BrPC}$ LUVs at different ionic strengths ranging from 100 to $1000 \mathrm{mM} \mathrm{NaCl}$ (Fig. 6). The increase in $\mathrm{NaCl}$ concentration dramatically decreased the quenching effect on $\operatorname{Trp}$ Fluorescence by $\mathrm{Br}$ atoms, regardless of the residues position. This can be explained by the masking effect of charges that weakens electrostatic interactions and reduce IFABP affinity for LUVs. This further supports the notion that electrostatic forces are essential for IFABP-membrane interactions. A similar effect was reported before for the non-collisional LFABP, for which an increase from 0 to $200 \mathrm{mM} \mathrm{NaCl}$ abolished completely its interaction with anionic vesicles [33].

\section{Discussion}

The results presented here provide new insights into FABPmembrane studies that may be relevant for understanding their specific functions and, eventually, for the therapeutic modulation of intestinal FABP's functions in diabetes and obesity. The acrylamide fluorescence quenching experiments indicated that IFABP portal region is partially protected by interaction with the lipid bilayer; while the quenching efficiency of brominated lipids on IFABP Trp mutants fluorescence indicate that the protein penetrates partially into the bilayer. Trp residues located in the portal region would be immersed between the polar head groups and the middle of the bilayer, in close contact with its hydrophobic core, depending on the phospholipid composition. In addition, the natural Trp residues ( 6 and 82 ) do not show a significant quenching by any of the brominated lipids used, which would suggest that these residues do not interact with the lipid phase, or at least do not come in close contact with the hydrophobic core of the membrane during the formation of the protein-membrane complex.

Previous studies, conducted to analyze FABP-membrane interactions have proved extensively that "collisional" FABPs can interact with phospholipid model membranes and that electrostatic interactions have a central role in the initial steps of the interaction $[10,30,34]$. Simulations have even suggested different modes of interaction for different FABPs [11] and a possible mechanistic model for the ligand download to the acceptor membranes starting from a configuration of IFABP on the surface (outside) of a model membrane [35]. However, this is the first time, to our knowledge, that the penetration depth of these soluble proteins into phospholipid membranes is systematically assessed experimentally, showing that apo-IFABP could penetrate into the phospholipid bilayer, as originally suggested by hydrophobic photolabeling experiments $[10,12]$. The photolabeling experiments have also shown that the interaction of IFABP with membranes is composition-dependent and increases with the negative charge of the membranes [12]. This is compatible with IFABPs proposed ligand

Table 4

Distribution analysis of Trp mutants of IFABP by brominated phospholipid quenching.

\begin{tabular}{|c|c|c|c|c|c|c|c|c|c|}
\hline \multirow[t]{2}{*}{ Fitting parameters ${ }^{\mathrm{a}}$} & \multicolumn{3}{|l|}{$100 \% \mathrm{BrPC}$} & \multicolumn{3}{|c|}{$75 \% \mathrm{BrPC}+25 \% \mathrm{EPC}$} & \multicolumn{3}{|c|}{$75 \% \mathrm{BrPC}+25 \% \mathrm{CL}$} \\
\hline & $h_{\mathrm{m}}(\AA)$ & $\sigma$ & $S$ & $h_{\mathrm{m}}(\AA)$ & $\sigma$ & $S$ & $h_{\mathrm{m}}(\AA)$ & $\sigma$ & $S$ \\
\hline F17W & $21.4 \pm 0.7$ & $10.8 \pm 0.5$ & $10.2 \pm 0.2$ & $15.1 \pm 1.0$ & $11.3 \pm 0.1$ & $3.3 \pm 0.4$ & $8.2 \pm 0.1$ & $7.2 \pm 0.4$ & $1.1 \pm 0.1$ \\
\hline L30W & $9.4 \pm 0.1$ & $3.6 \pm 0.8$ & $1.9 \pm 0.3$ & $9.4 \pm 0.2$ & $4.3 \pm 0.3$ & $1.0 \pm 0.2$ & $7.3 \pm 0.1$ & $4.1 \pm 0.2$ & $0.8 \pm 0.1$ \\
\hline F55W & $19.2 \pm 0.7$ & $10.0 \pm 0.3$ & $9.3 \pm 0.3$ & $10.4 \pm 0.4$ & $7.2 \pm 0.4$ & $1.8 \pm 0.3$ & $10.9 \pm 0.5$ & $4.6 \pm 0.5$ & $0.7 \pm 0.1$ \\
\hline I114W & NA & NA & NA & $15.7 \pm 1.0$ & $13.0 \pm 0.3$ & $2.5 \pm 0.3$ & $8.4 \pm 0.1$ & $2.4 \pm 0.1$ & $0.6 \pm 0.1$ \\
\hline
\end{tabular}

${ }^{a}$ Eq. (4) was fitted to the quenching of Trp mutants employing the distances of 10.8, 8.3 and 6.3 $\AA$ for the average position of 6,7-BrPC, 9,10-BrPC, and 11,12-BrPC quenching groups,

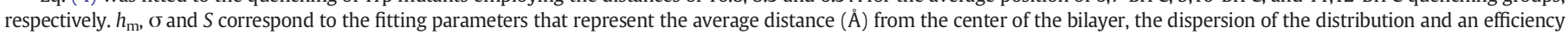
parameter of the Trp fluorescence quenching. Results are mean \pm SEM, N = 3 for all measurements, except for I114W with 25\% EPC LUVs (N = 2). NA: Not Applicable. 

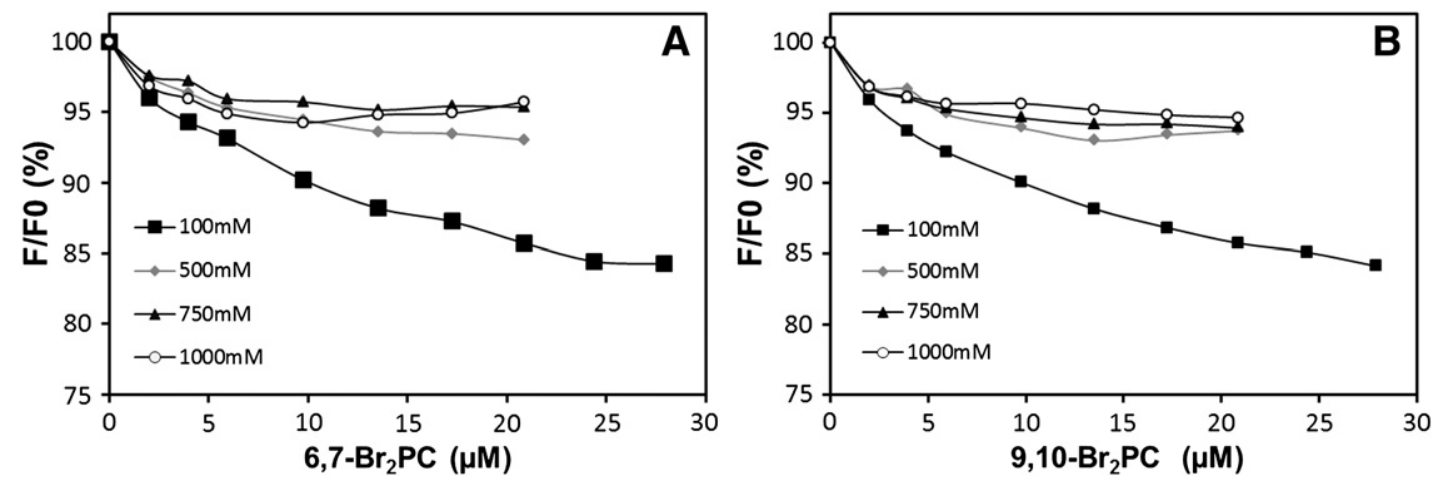

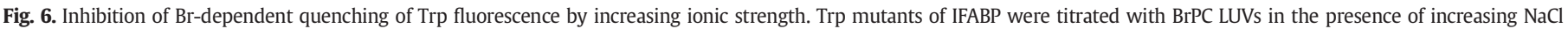
concentration (100-1000 mM). Panel A and B shows the results for L30W mutant for two different BrPC lipids with Br atoms at positions 6,7 and 9,10, respectively.

transfer mechanisms, which is collision-mediated and dependent on the composition of the phospholipid membranes. This interaction, although transient, may be critical for the functions of the FABPs in general and not only for IFABP, since several members of the family are also thought to effectively interact with membranes [36]. Furthermore, membrane insertion of the portal region may be associated with minor, but significant, conformational local changes in the portal region and the binding site that may induce and/or facilitate both ligand upload and release, avoiding the low probable ligand release into the aqueous medium. Furthermore, the differential protein configuration in the phospholipid bilayer depending on its lipid composition could be the key for selective intracellular trafficking of ligands. The models represented in Fig. 7, based on the distance restrictions estimated by the distribution analysis (Section 3.5), suggest a significant change in the orientation of IFABP during membrane interaction between POPC and CL-containing vesicles. This hypothesis urges to reanalyze by molecular dynamics the release of fatty acids from IFABP starting from the different configurations in which the protein is partially immersed in for each type of membrane.

Similar studies were performed already in other members of the FABP family, like Liver FABP (LFABP), where membrane association was observed only under conditions of low ionic strength [33]. In this case, two portal Trp mutants at positions 28 and 74 (L28W and $\mathrm{M} 74 \mathrm{~W}$ ) were employed in the analysis. The authors concluded, based on the selectivity of LFABP, that the large quenching effect observed $(>75 \%)$ was due to the insertion of one brominated acyl chain into the site 2 of the binding cavity, rather than due to the protein immersion into the membrane.

The membrane interaction of the residues of the portal region of IFABP is also consistent with previous works in which the centrality of the $\alpha$-helical region, particularly $\alpha$ II-helix, has been highlighted as part of the membrane binding domain and in determining the ligand transfer mechanism [6,37]. Total removal of the $\alpha$-helical domain undoubtedly impaired IFABP interaction with membranes and its ligand transfer capacity $[8,12]$. Moreover, photocrosslinking experiments followed by controlled proteolysis showed a markedly enrichment of the radiolabel in the peptide containing the $\alpha$-helical region of IFABP [12]. Compared to previous studies, the present work incorporates the participation of residues external to the helical region: from the $\beta C-\beta D$ loop, that is also known to participate in the portal domain that would control ligand entry and exit from the binding cavity [3]. But also a significant immersion of the $\operatorname{Trp}^{114}$ inserted in the BI-sheet was detected for anionic vesicles containing $25 \%$ CL. This possibility has been previously suggested for "diffusional" FABPs, like LFABP, LbFABP or CRBP, where the $\beta$-barrel presents the most probable domain for interaction with membranes $[11,38]$.

\section{Conclusion}

The proposed model of ligand transfer from IFABP to acceptor vesicles is believed to have different steps $[2,3,10]$. The initial interaction between the holo-protein and the membrane would be driven mainly

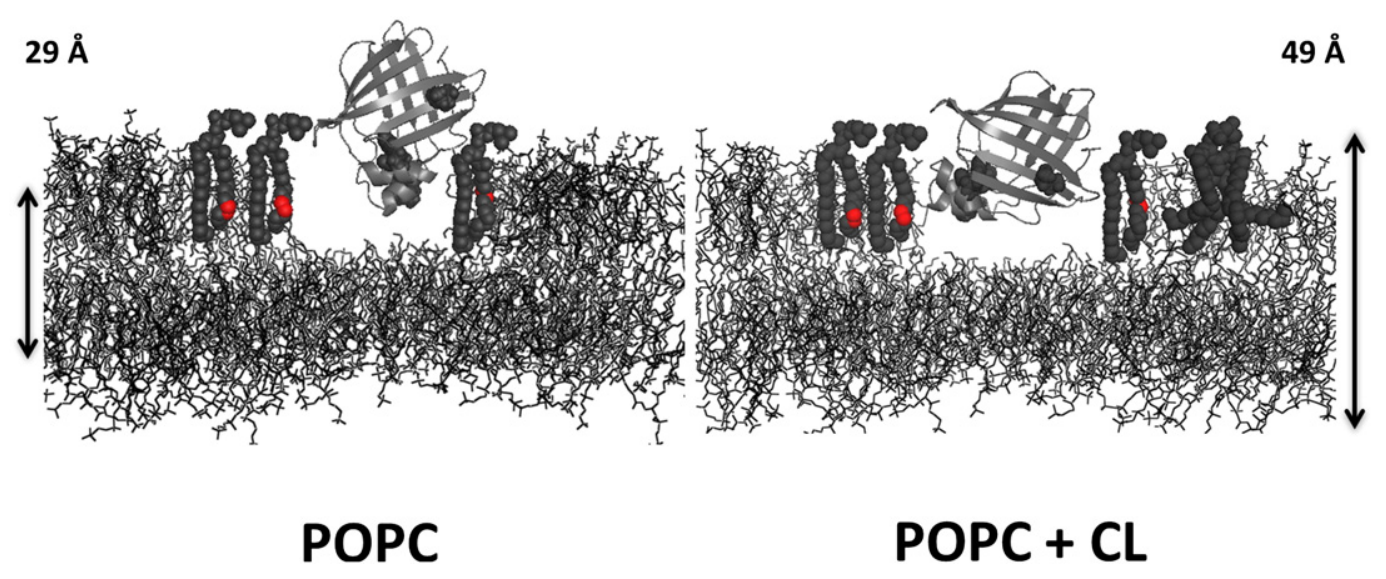

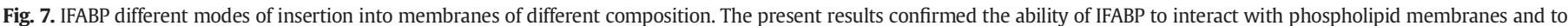

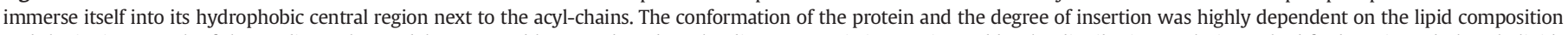

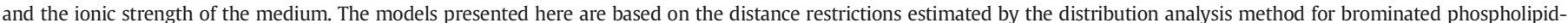

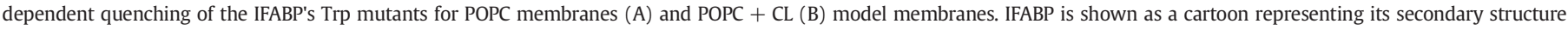

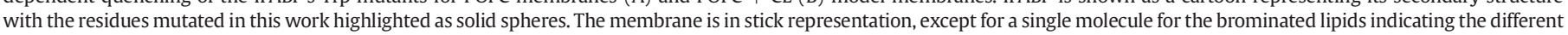
positions of the $\mathrm{Br}$ atoms, and one $\mathrm{CL}$ molecule, all in solid spheres representation. 
by electrostatic interactions; followed by a conformational transition of the protein from a closed to an open state. We have demonstrated here that part of the protein is immersed into the phospholipid membrane during the formation of the protein-membrane complex, and that this intermediate configuration may be different according to the lipid composition, particularly in the case of CL-containing membranes, like in the mitochondria. The conformational change of IFABP partially immersed into the phospholipid bilayer would promote the dissociation and exit of the fatty acid from the binding cavity to be incorporated into the membrane or to be taken by enzymes located in it. The conformational change that would promote the transfer of the ligand may involve both ionic and hydrophobic interactions with the membrane [10,31], but further studies are needed to understand how membrane interaction and conformational changes in the portal region are being translated in the exit/entry of the ligand to/from the phospholipid bilayer. An alternative was suggested by the analysis of LFABP-vesicles interaction, where a single acyl chain from a phospholipid of the membrane is believed to be inserted into the ligand binding cavity via the portal region, potentially promoting the release/upload of ligands [33].

One must also consider the relevance of the human Ala54Thr polymorphism of IFABP, present in the $\beta C-\beta D$ loop next to Phe ${ }^{55}$, which has been correlated with higher risk of developing insulin resistance and hypercholesterolemia in humans $[39,40]$ and with impaired $\Delta 6$ desaturase activity in obese children [41]. This polymorphism was reported to show a two-fold difference in affinity compared to the native protein, due to a new $\mathrm{H}$-bond bridge between the $\beta C-\beta D$ loop and $\alpha-\mathrm{II}$ helix that compromised the flexibility of the portal region and the entry of the fatty acids [42]. Localizing this residue deep in the membrane during the IFABP-membrane complex opens new possibilities for this polymorphism to affect the normal function of IFABP that must be further investigated and may account for the clinical observations.

\section{Acknowledgments}

This work was supported by ANPCyT (Argentina) through grants PICT-2004-26218 and PICT-2010-0506 to BC. EDG and LRS are also grateful to CONICET for their fellowships. LJFL also acknowledges support from the Alexander von Humboldt Foundation (Germany).

\section{References}

[1] J.F.C. Glatz, G.J. vanderVusse, Cellular fatty acid-binding proteins: their function and physiological significance, Prog. Lipid Res. 35 (1996) 243-282.

[2] B. Corsico, D.P. Cistola, C. Frieden, J. Storch, The helical domain of intestinal fatty acid binding protein is critical for collisional transfer of fatty acids to phospholipid membranes, Proc. Natl. Acad. Sci. U. S. A. 95 (1998) 12174-12178.

[3] M.E. Hodsdon, D.P. Cistola, Discrete backbone disorder in the nuclear magnetic resonance structure of apo intestinal fatty acid-binding protein: Implications for the mechanism of ligand entry, Biochemistry 36 (1997) 1450-1460.

[4] N.M. Bass, Function and regulation of hepatic and intestinal fatty-acid bindingproteins, Chem. Phys. Lipids 38 (1985) 95-114.

[5] K.T. Hsu, J. Storch, Fatty acid transfer from liver and intestinal fatty acid-binding proteins to membranes occurs by different mechanisms, J. Biol. Chem. 271 (1996) 13317-13323.

[6] B. Corsico, H.L. Liou, J. Storch, The alpha-helical domain of liver fatty acid binding protein is responsible for the diffusion-mediated transfer of fatty acids to phospholipid membranes, Biochemistry 43 (2004) 3600-3607.

[7] H.K. Kim, J. Storch, Free fatty-acid transfer from rat-liver fatty acid-binding protein to phospholipid-vesicles - effect of ligand and solution properties, J. Biol. Chem. 267 (1992) 77-82.

[8] F. Wu, B. Corsico, C.R. Flach, D.P. Cistola, J. Storch, R. Mendelsohn, Deletion of the helical motif in the intestinal fatty acid-binding protein reduces its interactions with membrane monolayers: Brewster angle microscopy, IR reflection-absorption spectroscopy, and surface pressure studies, Biochemistry 40 (2001) 1976-1983.

[9] K. Kim, D.P. Cistola, C. Frieden, Intestinal fatty acid-binding protein: the structure and stability of a helix-less variant, Biochemistry 35 (1996) 7553-7558.

[10] L.J. Falomir Lockhart, L. Laborde, P.C. Kahn, J. Storch, B. Corsico, Protein-membrane interaction and fatty acid transfer from intestinal fatty acid-binding protein to membranes - support for a multistep process, J. Biol. Chem. 281 (2006) 13979-13989.

[11] F. Zamarreno, F.E. Herrera, B. Corsico, M.D. Costabel, Similar structures but different mechanisms Prediction of FABPs-membrane interaction by electrostatic calculation, Biochim. Biophys. Acta Biomembr. 1818 (2012) 1691-1697.
[12] L.J. Falomir-Lockhart, G.R. Franchini, M. Ximena Guerbi, J. Storch, B. Corsico, Interaction of enterocyte FABPs with phospholipid membranes: clues for specific physiological roles, Biochim. Biophys. Acta Mol. Cell Biol. Lipids 1811 (2011) 452-459.

[13] J. Storch, B. Corsico, The emerging functions and mechanisms of mammalian fatty acid-binding proteins, Annu. Rev. Nutr. 28 (2008) 73-95.

[14] M. Kammann, J. Laufs, J. Schell, B. Gronenborn, Rapid insertional mutagenesis of DNA by Polymerase Chain-Reaction (PCR), Nucleic Acids Res. 17 (1989) 5404

[15] S.C. Gill, P.H. Vonhippel, Calculation of protein extinction coefficients from amino-acid sequence data, Anal. Biochem. 182 (1989) 319-326.

[16] C. Huang, T.E. Thompson, Preparation of homogeneous, single-walled phosphatidylcholine vesicles, Methods Enzymol. 32 (1974) 5.

[17] J. Storch, A.M. Kleinfeld, N.M. Bass, H. Shields, Interaction of fatty-acid binding-protein (FABP) with fluorescent analogs of free fatty-acids, Biophys. J. 49 (1986) A106.

[18] L. Whitmore, B.A. Wallace, Protein secondary structure analyses from circular dichroism spectroscopy: methods and reference databases, Biopolymers 89 (2008) 392-400.

[19] J.B. Massey, D.H. Bick, H.J. Pownall, Spontaneous transfer of monoacyl amphiphiles between lipid and protein surfaces, Biophys. J. 72 (1997) 1732-1743.

[20] J. Storch, N.M. Bass, Transfer of fluorescent fatty-acids from liver and heart fatty acid-binding proteins to model membranes, J. Biol. Chem. 265 (1990) 7827-7831.

[21] F.S. Abrams, E. London, Calibration of the parallax fluorescence quenching method for determination of membrane penetration depth - refinement and comparison of quenching by spin-labeled and brominated lipids, Biochemistry 31 (1992) 5312-5322.

[22] T.J. McIntosh, P.W. Holloway, Determination of the depth of bromine atoms in bilayers formed from bromolipid probes, Biochemistry 26 (1987) 1783-1788.

[23] A.S. Ladokhin, Analysis of protein and peptide penetration into membranes by depth-dependent fluorescence quenching: theoretical considerations, Biophys. J. 76 (1999) 946-955.

[24] D. Van Der Spoel, E. Lindahl, B. Hess, G. Groenhof, A.E. Mark, H.J. Berendsen, GROMACS: fast, flexible, and free, J. Comput. Chem. 26 (2005) 1701-1718.

[25] PyMOL Molecular Graphics System, Schrödinger, LLC, 15.04.2013. www.pymol.org.

[26] E.M. Clerico, M.R. Ermacora, Tryptophan mutants of intestinal fatty acid-binding protein: ultraviolet absorption and circular dichroism studies, Arch. Biochem. Biophys. 395 (2001) 215-224.

[27] J.C. Sacchettini, J.I. Gordon, L.J. Banaszak, Refined apoprotein structure of rat intestinal fatty-acid binding-protein produced in Escherichia coli, Proc. Natl. Acad. Sci. U. S. A. 86 (1989) 7736-7740.

[28] J.C. Sacchettini, J.I. Gordon, L.J. Banaszak, Crystal structure of rat intestinal fatty-acid-binding protein. Refinement and analysis of the Escherichia coli-derived protein with bound palmitate, J. Mol. Biol. 208 (1989) 327-339.

[29] T. Markello, A. Zlotnick, J. Everett, J. Tennyson, P.W. Holloway, Determination of the topography of cytochrome-b5 in lipid vesicles by fluorescence quenching, Biochemistry 24 (1985) 2895-2901.

[30] P.E. Thoren, D. Persson, E.K. Esbjorner, M. Goksor, P. Lincoln, B. Norden, Membrane binding and translocation of cell-penetrating peptides, Biochemistry 43 (2004) 3471-3489.

[31] B. Corsico, G.R. Franchini, K.T. Hsu, J. Storch, Fatty acid transfer from intestinal fatty acid binding protein to membranes: electrostatic and hydrophobic interactions, J. Lipid Res. 46 (2005) 1765-1772.

[32] S.R. Cheruku, Z. Xu, R. Dutia, P. Lobel, J. Storch, Mechanism of cholesterol transfer from the Niemann-Pick type C2 protein to model membranes supports a role in lysosomal cholesterol transport, J. Biol. Chem. 281 (2006) 31594-31604.

[33] R.M. Hagan, J. Worner-Gibbs, D.C. Wilton, The interaction of liver fatty-acid-binding protein (FABP) with anionic phospholipid vesicles: is there extended phospholipid anchorage under these conditions? Biochem. J. 410 (2008) 123-129.

[34] H.K. Kim, J. Storch, Mechanism of free fatty-acid transfer from rat-heart fatty acid-binding protein to phospholipid-membranes - evidence for a collisional process, J. Biol. Chem. 267 (1992) 20051-20056.

[35] M. Mihajlovic, T. Lazaridis, Modeling fatty acid delivery from intestinal fatty acid binding protein to a membrane, Protein Sci. 16 (2007) 2042-2055.

[36] B. Corsico, Intracellular lipid transport: structure-function relationships in fatty acid binding proteins, Futur. Lipidol. 1 (2006) 615-622.

[37] G.R. Franchini, J. Storch, B. Corsico, The integrity of the alpha-helical domain of intestinal fatty acid binding protein is essential for the collision-mediated transfer of fatty acids to phospholipid membranes, Biochim. Biophys. Acta Mol. Cell Biol. Lipids 1781 (2008) 192-199.

[38] S.M. Di Pietro, B. Corsico, M. Perduca, H.L. Monaco, J.A. Santome, Structural and biochemical characterization of toad liver fatty acid-binding protein, Biochemistry 42 (2003) 8192-8203.

[39] R.E. Pratley, L. Baier, D.A. Pan, A.D. Salbe, L. Storlien, E. Ravussin, C. Bogardus, Effects of an Ala54Thr polymorphism in the intestinal fatty acid-binding protein on responses to dietary fat in humans, J. Lipid Res. 41 (2000) 2002-2008.

[40] S. Bertolini, L. Pisciotta, L. Di Scala, S. Langheim, A. Bellocchio, P. Masturzo, A. Cantafora, S. Martini, M. Averna, G. Pes, C. Stefanutti, S. Calandra, Genetic polymorphisms affecting the phenotypic expression of familial hypercholesterolemia, Atherosclerosis 174 (2004) 57-65.

[41] T. Okada, N.F. Sato, Y. Kuromori, M. Miyashita, F. Iwata, M. Hara, K. Harada, H. Hattori, Thr-encoding allele homozygosity at codon 54 of FABP 2 gene may be associated with impaired delta 6 desatruase activity and reduced plasma arachidonic acid in obese children, J. Atheroscler. Thromb. 13 (2006) 192-196.

[42] F.L. Zhang, C. Lucke, L.J. Baier, J.C. Sacchettini, J.A. Hamilton, Solution structure of human intestinal fatty acid binding protein with a naturally-occurring single amino acid substitution (A54T) that is associated with altered lipid metabolism, Biochemistry 42 (2003) 7339-7347. 